\title{
Die Myofilamente glatter Muskulatur und ihr Verhältnis zu den Myofilamenten quergestreifter Muskulatur. Eine elektronen- mikroskopische Studie an Muskelzellen der Weinbergschnecke
}

\author{
Von Friedrich-Wilhelm Schlote* \\ Aus dem Zoologischen Institut der Universität Göttingen \\ (Z. Naturforschg. 12 b, 647-653 [1957] ; eingegangen am 1. Juli 1957)
}

\begin{abstract}
Die Muskelzellen eines glatten Muskels der Weinbergschnecke grenzen mit Sarkolemm und Basalmembran gegen die kollagenführende, Fibroblasten enthaltende Grundsubstanz des Bindegewebes. Die Peripherie ihres Sarkoplasmas enthält Röhrchen des sarkoplasmatischen Retikulums von etwa $300 \AA$ A Durchmesser, ferner großlumige Vakuolen mit sehr geringer Elektronendichte und wenige Mitochondrien. Das Innere der Muskelzelle wird von Myofilamenten eingenommen. Sie liegen anscheinend regellos nebeneinander. Längsschnitte durch Filamentgruppen zeigen eine Querstreifung, die mit $220 \AA ̊$ etwa jener der quergestreiften Skelettmuskulatur entspricht. Die Masse der Filamente ist $190 \AA$ dick. Daneben kommen dünnere Myofilamente von etwa $130 \AA ̊$ Durchmesser vor. An Hand dieses Befundes werden die bisher bekannt gewordenen Myofilament-Dicken der Literatur miteinander verglichen. Man erhält für ihre Durchmesser eine geometrische Reihe mit der Steigung 2. Sie beginnt bei Filamenten von etwa 30 Å Dicke und endet bei Filamenten von etwa $260 \AA \AA$ Dicke. Dieser überraschende Befund, in den sich die Filamentdicke von $190 \AA \AA$ aus dem hier untersuchten Muskel mit einer plausiblen Zusatzannahme einfügen läßt, wird auf eine Reihe von möglichen Konsequenzen hin ausführlich diskutiert und als Argument für eine von Muskelart und Kontraktionszustand abhängige schrittweise Aufschraubung dünnster Filamenteinheiten angesehen.
\end{abstract}

Glatte Muskelzellen kommen in einer Reihe von Modifikationen bei sehr vielen niederen Tieren vor. Bei Mollusken, Anneliden, Nemathelminten, Plathelminten bis herab zu den Coelenteraten beherrschen sie das Bild. Die Wirbeltiere besitzen sie in ihrem Verdauungstrakt. Den Arthropoden scheinen sie völlig zu fehlen ${ }^{1}$ (zit. WiggLesworth 1953).

Uber ihre Physiologie ist eine Menge bekannt ${ }^{2,3}$ (Postma 1951, Bozler 1948), jedoch so widerspruchsvoll, daß es schwer wird, ein einheitliches Bild zu entwerfen ${ }^{4}$ (Zusammenfassung bei BRECHT 1950). Extrakte glatter Muskulatur von Anodonta (Teichmuschel) verhalten sich im Modellversuch wie die von quergestreifter Muskulatur ${ }^{5}$ (Ulbrecht 1952). Die Histologie ist bei den Wirbeltieren gründlich untersucht 6,6a (s. z. B. BargmanN 1956, HäGGQvist 1956), bei den Wirbellosen mehr in großen Zü-

* Die Arbeit wurde durchgeführt mit Unterstützung der Deutschen Forschungsgemeins chaft und der Göttinger Akademie der Wissen$\mathrm{s} \mathrm{c}$ h a f t e n. Herrn Dr. J. A. NúÑ̃z möchte ich danken für das Überlassen einiger Bilder, Frl. Gisela Föge für unermüdliche Hilfe.

1 V. B. Wigglesworth, The Principles of Insect Physiology. 5. Aufl., Methuen and Co., London 1953.

2 N. Postma, Extr. d'Arch. Neerland d'Zoolog. 8, 374 [1951].

3 E. Bozler, Experientia [Basel] 4, 213 [1948].

${ }^{4}$ K. Brecht, Fortschr. Zool. (M. Hartmans), 9, 500 [1945 bis 1950].

5 G. Ulbrecht u. M. Ulbrecht, Z. Naturforschg. 7 b, 434 [1952].

6 W. Bargmann, Histologische und mikroskopische Anatomie des Menschen. 2. Aufl., Georg Thieme, Stuttgart 1956. gen bekannt ${ }^{7}$ (Übersicht bei Hartmann 1953). Zur submikroskopischen Morphologie glatter Muskulatur liegen erst wenige Befunde röntgenographischer ${ }^{8,9}$ (BEAR 1945, SсHмitt et al. 1947) und übermikroskopischer Art 10-12 (Hodge et al. 1954, GANSLER 1956, Hanson 1956) vor.

Es wäre denkbar, daß in glatter Muskulatur der Bau der kontraktilen Elemente ursprünglicher und daher der submorphologische Ablauf einer Kontraktion einfacher zu verstehen ist als in der hochspezialisierten quergestreiften Muskulatur von Arthropoden und Vertebraten. Im folgenden wird an Hand erster Befunde an Muskeln der Weinbergschnecke deshalb zunächst ein Überblick über den Feinbau glatter Muskelzellen gegeben werden. Die dabei auftretenden zahlreichen Einzelfragen sind später getrennt zu analysieren.

6a G. HäGgQvist, Die Gewebe, 4.Teil: „Gewebe u. Systeme d. Muskulatur" in: Handb. d. mikrosk. Anatomie d. Menschen 2, Teil 3, Suppl.; Springer, Berlin 1956.

7 M. Hartmann, Allgemeine Biologie. 4. Aufl., Gustav Fischer, Stuttgart 1953.

8 R. S. BEAR, J. Amer. chem. Soc. 67, 1625 [1945].

9 F. O. Schmitt, R. S. Bear, C. E. Hall u. M. A. Jakus, Ann. New York Acad. Sci. 47, 799 [1947].

10 J. A. Hodge, H. E. Huxley u. D. Spiro, J. exp. Medicine 99, 201 [1954].

11 H. Gansler, Electron Microscopy, Proc. Stockholm Conf. 210, Almqvist and Wiksell, Stockholm 1956 sowie mündliche Mitteilung.

12 J. Hanson, J. Biophysic. and Biochem. Cytol. 3, 111 [1957]. 


\section{Methodisches}

Fixierung teilkontrahierter Penisretraktor-Muskeln aus über Nacht vorgekühlten Weinbergschnecken in isotonischer gepufferter 1-proz. $\mathrm{OsO}_{4}$-Lösung bei $+6^{\circ} \mathrm{C}$. Dehydrierung, Einbettung und Dünnschnittechnik nach den Standardverfahren. Elektronenmikroskop Siemens Modell 1939, jedoch ${ }^{13}$ nach Schlote (1957) mit einer Vorrichtung zum Herstellen von Durchfokus-Aufnahmen versehen. Fototechnischer Film F02. Entwickler Perutz OP 106. Übermikroskopische Vergrößerung bis 9000 fach.

\section{Befunde}

Die Muskeln von Helix pomatia setzen sich aus langen, an ihrem Ende leicht zugespitzten glatten Muskelzellen zusammen. In Herz und Schlundkopf sollen sie während mancher Funktionszustände eine primitive Art der Querstreifung besitzen ${ }^{14}$ (PLENK 1924). Ob es sich dabei um intrazelluläre Querscheiben ähnlich den Z-Scheiben von Skelettmuskeln handelt oder nur eine unregelmäßige Einfaltung der Zelloberfläche vorliegt, ist bisher nicht entschieden.

Nur ein Teil der Muskeln einer Weinbergschnecke ist anatomisch soweit definiert, daß er in aufeinanderfolgenden Präparationen unschwer wiedergefunden werden kann. Hierzu gehört der Penisretraktor. Er läßt sich, weil dünn, leicht total in situ fixieren. Im gedehnten Zustand ist er - reversibel - mindestens $24 \mathrm{~mm}$ lang, in kontrahiertem Zustand etwa $3 \mathrm{~mm}$. Das Verhältnis zwischen kleinster und größter Länge beträgt also mindestens $1: 8$ (!). Zwischen seinen Muskelzellen liegen Fibroblasten (Abb. $1^{*}$ ). Beide, Muskelzellen und Fibroblasten, sind in kollagenhaltige Grundsubstanz eingebettet. Gegen die Leibeshöhle ist der Muskel durch eine dichtere Schicht von Kollagen und Fibroblasten abgegrenzt. Außerdem enthält er noch andere Zellarten, so z. B. großlumige, verzweigte Zellen, deren Plasma sich nach Alkohol-Eisessig-Fixierung mit Pyronin intensiv rötet. In ihrem Inneren können mit Hämatoxylin stark färbbare Grana wechselnder Größe und Form liegen.

Lichtmikroskopisch besitzt jede Muskelzelle an ihrer Peripherie eine dünne Zone, die sich nach Bouin-Allen-Fixierung mit Säurefuchsin (MAsson) rot färbt. Alle zentraler gelegenen Teile werden,

\footnotetext{
${ }^{13}$ Fr. W. Schlote, Z. wiss. Mikroskopie, mikroskop. Techn. 1957, im Druck.

14 H. PLENK, Z. wiss. Zool. 122, 1 [1924].
}

sieht man von den Bereichen aus der Nähe des Zellkernes ab, nach dieser Methode homogen blaugrau.

Übermikroskopisch ist die Peripherie der Muskelzelle verhältnismäßig licht. Sie wird schütter durchsetzt von Röhrchen, die einen Außendurchmesser von etwa $300 \AA$ besitzen (Abb. 2). In unmittelbarer Nähe der Zellgrenzmembran, dem Sarkolemm, gelegentlich aber auch zwischen den Myofilamenten sowie in Kernnähe (Abb. 7) finden sich eine Anzahl von Vakuolen, welche durch eine scharf kontrastierende Schicht gegen das Sarkoplasma abgegrenzt sind. Die Elektronendichte ihres Lumens ist etwa so groß wie oder noch kleiner als die der kollagenführenden Grundsubstanz. Sie steigt zur Peripherie der Vakuole hin leicht an. Das Sarkolemm und die es begleitende Basalmembran verlaufen häufig so, als ob das Sarkoplasma amöboide Fortsätze in die Grundsubstanz des Bindegewebes aussenden könnte (Abb. 2).

Der Hauptteil einer glatten Muskelzelle wird von den Myofilamenten eingenommen. Nach ersten Messungen an teilkontrahierten Muskeln enthält ein Querschnitt 400-600 Myofilamente pro $\mu^{2}$. Aus mehreren Gründen unterliegt diese Zahl einer verhältnismäßig großen Streuung. Einmal sind die Fäden weder ganz gleichmäßig verteilt noch in bisher erkennbarer Weise kristalloid geordnet. Vielmehr liegen sie teils scheinbar willkürlich nebeneinander, teils lassen sie dann und wann eine gewisse Gruppierung erkennen (Abb. 5,6). Da aber auch schlechte Fixierung und Schrumpfung eine Bündelung von Myofilamenten hervorrufen kann, ist bei der Deutung Vorsicht geboten.

Sodann finden sich im Inneren einer Muskelzelle zwischen den Myofilamenten hellere plasmatische Zonen, die Mitochondrien enthalten können (Abb. 6), außerdem gelegentlich jene oben beschriebenen hellen Vakuolen sowie sarkoplasmatisches Retikulum. Schließlich zeigt jeder Querschnitt hier und da zwischen den Myofilamenten kreisförmige elektronendichte Massen vom mehrfachen Durchmesser eines Myofilamentes (Abb. 4).

Nicht alle Filamente eines Zellquerschnittes sind gleich dick (Abb. 5, 6). Seinerzeit wurde der Durchmesser der damals fälschlich „Fibrillen“ genannten Myofilamente der glatten Muskelzellen aus dem Perineurium von Helix mit $280 \AA$ angegeben ${ }^{15}$

* Abb. $1-7$ s. Tafel S. $644 \mathrm{f}-\mathrm{h}$.

15 Fr. W. Schlote, Z. Zellforsch. mikroskop. Anatom. 45, 543 [1956]. 
(Schlote 1956). Gelegentlich finden sich auch hier im teilkontrahierten Penisretraktormuskel Zellen, die Myofilamente etwa dieser Dicke besitzen. Die überwiegende Zahl der Zellen enthält jedoch Fäden, die einem anderen Kollektiv angehören.. Wir haben über 1000 Querschnitte von Myofilamenten ausgemessen. Ihr Durchmesser läßt sich auch auf Schrägschnitten eindeutig bestimmen, da schräge Schnitte durch Zylinder Ellipsen darstellen, deren kleine Achse dem Zylinderdurchmesser gleicht. Werden im Rahmen unserer Meßgenauigkeit Größenklassen gebildet, so erhält man eine Kurve, die zu den niederen Größenklassen hin einen zweiten Gipfel bei etwa $130 \AA$ aufweist. Das Hauptmaximum liegt bei $190 \AA$. Darüber hinaus fällt die Kurve wie eine echte $\mathrm{Zu}$ fallskurve ab (Abb. 8).

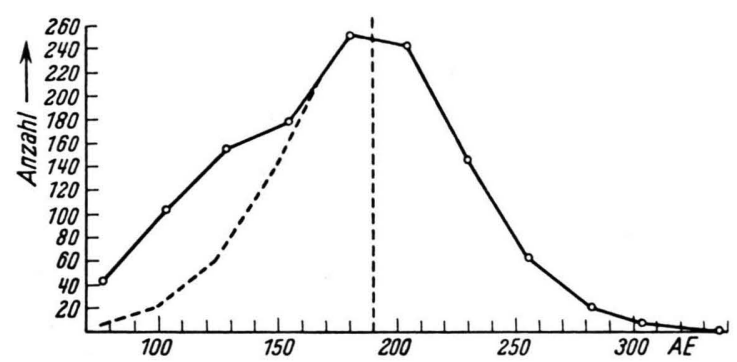

Abb. 8. Myofilamentdicken in glatten Muskelzellen von Helix. Die Filamente wurden, auf etwa $5-10 \mathrm{~mm}$ nachvergrößert, auf Papier projiziert, gezeichnet, ausgemessen und Größenklassen von 0,5 zu $0,5 \mathrm{~mm}$ gebildet. Die Ordinate gibt die Anzahl der für jede Größenklasse gefundenen Myofilamente an. Abszisse: Filament-Durchmesser. Die rechte Seite der Kurve ist, an der 190 Å-Ordinate gespiegelt, auf der linken Seite gestrichelt eingetragen, um das 2. Maximum bei etwa $130 \AA$ Å deutlicher zu machen.

Versuche, den Feinbau der Filamente darzustellen, sind bisher fehlgeschlagen. Ohne Kontrastierung mit Phosphorwolframsäure scheint die Muskelzelle, sieht man von Mitochondrien, Röhrchen und Vakuolen ab, innen praktisch völlig homogen $\mathrm{zu}$ sein. Leichte Kontrastierung mit Phosphorwolframsäure $(0,5 \%$ in 70 -proz. Alkohol für 1 Stde.) hebt die Myofilamente etwas hervor. An sehr dünnen Querschnitten gewinnt man den Eindruck, als ob jedes Filament aus einer verhältnismäßig geringen Anzahl von Untereinheiten aufgebaut sei. Dieser Befund ist noch nicht deutlich genug, um dargestellt werden zu können. Noch stärkere Kontrastierung (1\% Phosphorwolframsäure in 70-proz. Alkohol für 2 Stdn.) läßt die Myofilamente dem flüchtigen Betrachter homogen dunkelgrau erscheinen. Bei ge- nauerem Zusehen erkennt man dann und wann eine Streifung im Filament-Querschnitt. Sie verläuft, kontrolliert man mehrere Myofilamente, in allen Koordinaten des Bildes und zeigt damit an, daß sie nichts mit dem Astigmatismus unseres Objektivs zu tun hat (Abb. 5). Weiter ist auf manchen Bildern die Randzone eines Myofilamentes dunkler als sein Inneres (Abb. 2, 4). Hier ist ein Teil der Filamente schräg getroffen. Wären sie in sich homogen, dann müßte jeder schwarze Punkt nach beiden Seiten in eine gleichmäßig verschwimmende graue Zone übergehen, je nachdem, wie lang die Projektion des schräg stehenden Filamentes auf die Schnittebene ist. Tatsächlich steckt aber jeder Punkt zwischen 2 dünnen schwarzen Linien, der Projektion des elektronendichten Filamentrandes auf die Schnittebene. Da die Möglichkeit besteht, daß es sich hierbei um ein von der Fixations- und Kontrastierdauer abhängiges Fortschreiten der Schwärzung von außen nach innen handelt, soll im Augenblick auf diese Erscheinung nicht näher eingegangen werden.

Zwischen den Myofilamenten sind auf manchen Querschnitten kurze dünne, mehr oder weniger in der Schnittebene verlaufende Fibrillen sichtbar (Abb. 5). Ihr Durchmesser beträgt etwa $60 \AA$. Die Parallelorientierung, die sie auf Bildern wie Abb. 5 zu besitzen scheinen, läßt sich z. T. auf den an unserem Gerät nicht korrigierbaren Astigmatismus in Verbindung mit leichter Unterfokussierung zurückführen.

Gelegentlich finden sich auf Quer- und Schrägschnitten auch Muskelzellen, deren Myofilamente nicht quer, sondern längs getroffen sind (Abb. 2, $3,4)$. Dort, wo über ein größeres Stück hin eine Anzahl von Filamenten wirklich der Länge nach angeschnitten wurde (Abb. 3, 4), wird eine Querstreifung sichtbar. Die Periode beträgt $220 \pm 30 \AA \quad(n=23$, einfacher mittlerer Fehler).

\section{- Diskussion}

An Hand der oben beschriebenen Befunde, durch die ein erster Einblick in den Feinbau der glatten Muskelzellen von Helix gewonnen wurde, soll nun die submikroskopische Morphologie der verschiedenen Muskelzellarten miteinander verglichen werden. Dies wird ermöglichen, hinsichtlich des morphologischen. Ablaufes der Kontraktion Alternativfragen zu stellen, welche einer für später geplanten submikroskopischen Untersuchung zugänglich sind. 


\section{a) Plasmastruktur}

Die Mitochondrienzahl in glatten Muskelzellen ist außerordentlich klein. Dies entspricht der geringen Leistung (Cal/sec, Watt), die solche Muskeln aufzubringen haben. Dafür finden sich größere Vakuolen. Die Elektronendichte ihres Lumens (Abb. 7) scheint z.T. noch unter jener der Grundsubstanz des Bindegewebes zu liegen. Vergleichende lichtmikroskopische Untersuchungen fehlen. Eine Zuordnung zu bestimmten Stoffen ist daher noch nicht möglich. Die Röhrchen von etwa $30 \AA$ Durchmesser, welche vor allem an der Peripherie der Zelle gefunden wurden (vgl. aber Abb. 6), dürften dem sarkoplasmatischen Retikulum quergestreifter Muskelfasern $^{16,17}$ (Porter 1956, Andersson 1956) gleichkommen.

\section{b) Anordnung der Myofilamente}

Die Myofilamente der Muskelzellen eines Penisretraktors von Helix haben einen wesentlich größeren Durchmesser als die Filamente quergestreifter Muskelfasern. Darüberhinaus sind sie, betrachtet man Querschnitte (Abb. 2, 4-7), ungleich dick. Zudem ist nirgends eine kristalloide Packung zu erkennen, wie sie in quergestreiften Muskelfibrillen so typisch in Erscheinung tritt. Vielmehr liegen die Filamente, allenfalls locker in Gruppen zusammengefaßt (Abb. 5, 6), ohne ersichtliche Ordnung im Inneren der Muskelzellen. Im hier untersuchten teilkontrahierten Zustand des ganzen Muskels verlaufen sie innerhalb ihrer Zellen nicht einmal alle einander parallel. Immer wieder findet man Zellen, in denen ganze Fadengruppen schräg bis quer zur Längsachse stehen (Abb. 2-4).

Dies dürfte folgenden Grund haben: der Penisretraktor einer frisch präparierten Schnecke ist gewöhnlich stark kontrahiert und dann etwa $3 \mathrm{~mm}$ lang. Zur Fixierung wird er in situ durch sanften Zug (gegen einigen Widerstand) bis auf etwa $15 \mathrm{~mm}$ gedehnt und an Ort und Stelle mit dem Fixiermittel übergossen. Seine wahre Länge ist jedoch größer. Noch nach einer Dehnung auf $24 \mathrm{~mm}$ kann er sich, erregt durch mechanische Reizung des Penis,

16 K. R. Ponter, J. Biophys. and Biochem. Cytol. 2, Suppl., 163 [1956].

17 E. Andersson, Electron Microscopy, Proc. Stockholm Conf. 208, Almqvist and Wiksell, Stockholm 1956.

18 F. S. Suöstrand u. E. Andersson, Exp. Cell. Res. 11, 493 [1956]. auf seine Ausgangsstellung $(3 \mathrm{~mm})$ zurück verkürzen. Der Abstand zwischen der Geschlechtsöffnung und dem Ansatzpunkt des Penisretraktors unter dem Lungenboden beträgt bei einer kriechenden Schnecke etwa $25 \mathrm{~mm}$. Da der Penis bei der Kopulation aus der Geschlechtsöffnung heraustritt, kommt ein Dehnungsverhältnis von $3: 24$ oder $1: 8$ etwa den physiologischen Verhältnissen gleich. Muskeln, die bei der Fixierung kürzer sind als ihre Maximallänge und das sind hier alle - , brauchen nicht ausschließlich aus kontrahierten Zellen zu bestehen. Sind vielmehr darunter Zellen, deren Myofilamente Schlangenlinien zur Längsachse der Zelle beschreiben, so können diese nicht kontrahiert sein. Sie müssen, als sich ein Teil der Zellen zusammenzog, passiv gestaucht worden sein.

Dieser Zustand, den man von quergestreiften Muskelfasern nicht kennt, ist für glatte Muskelzellen typisch. Er dürfte mit der Verteilung der nervösen Erregung im ganzen Muskel zusammenhängen.

\section{c) Vergleichende Betrachtung von Myofilamenten}

Besonderes Interesse verdienen die Myofilamente. Ein Vergleich der Angaben anderer Autoren mit den bei Helix gefundenen Werten bringt merkwürdige Verhältnisse zutage: SJöstrand und Andersson 18, 19 (1956, a, b) finden in stark gedehnten quergestreiften Muskeln des Frosches drei ein Filament aufbauende Fäden von je 10-20 ̊ Durchmesser. In der I-Zone mißt ein Myofilament bei ihnen etwa $40 \AA$ im Durchmesser. Die Filamente der A-Zone sind je nach Kontraktionszustand $60-140 \AA$ dick. Sie scheinen aus einem schraubig gewundenem, etwa $30 \AA$ dicken Faden aufgebaut zu sein. Sснмiтt et al. ${ }^{9}$ errechnen aus Kleinwinkel-Röntgendiagrammen für den Myosinanteil glatter Adductormuskeln verschiedener $\mathrm{Mu}-$ scheln eine Fadenstärke von $27 \AA$.

$\mathrm{GANSLER}^{11}$ sieht in der Uterusmuskulatur gravider Ratten Filamente von etwa $60 \AA$ Dicke. SpIro ${ }^{20}$ (1956) schreibt den Myofilamenten aus Herzen von Hühnerembryonen und wachsenden Krabbenmuskeln Dicken von 60 - $70 \AA \AA$ zu. Hodge ${ }^{21}$ (1956) und viele andere Autoren finden in der A-Zone quergestreifter

19 F. S. Suöstrand u. E. Andersson, Electron Microscopy, Proc. Stockholm Conf. 204, Almqvist and Wiksell, Stockholm 1956.

20 D. Sprro, J. Biophysic. and Biochem. Cytol. 2, Suppl., 167 [1956].

21 A. J. Hodge, J. Biophysic. and Biochem. Cytol. 2, Suppl., 131 [1956]. 
Muskelfibrillen Filamentquerschnitte von etwa 120 Å. Bei Helix liegen nebeneinander Filamente von etwa 130 und $190 \AA$ Durchmesser, in Muskelzellen des Perineuriums sogar solche von $280 \AA$ Durchmesser $^{15}$ (Schlote 1956). Hanson ${ }^{12}$ (1957) beschreibt aus glatten Muskelzellen des Regenwurmes Filamente von etwa $250 \AA$ Dicke bei großer Streuung *. Nimmt man an, daß alledem eine gemeinsame Gesetzmäßigkeit zugrunde liegt, dann lassen sich die Werte wie folgt ordnen: zellen enthalten ${ }^{14}$ (PLENK 1924). Quergestreifte Muskulatur dagegen wird von außen her innerviert. Auch der hier untersuchte Penisretraktor von Helix besitzt Zellen, die sich lichtmikroskopisch der Art ihrer Verzweigung nach mit Ganglienzellen vergleichen lassen. Elektronenmikroskopisch finden sich in ihm immer wieder Zellen, die eine Unmenge von Doppelmembranen im Plasma enthalten und mit Sicherheit keine Muskelzellen sind.

\begin{tabular}{|c|c|c|c|}
\hline Objekt & $\begin{array}{c}\text { Meßwert } \\
{[\AA]}\end{array}$ & Autor & $\begin{array}{c}\text { Sollwert } \\
{[\AA]}\end{array}$ \\
\hline Quergestreifte Muskeln, gedehnt, Frosch & $10-20$. & SJöstrand und ANDERSSON 18,19 & 16,3 \\
\hline $\begin{array}{l}\text { Myosin aus Adductoren von Muscheln } \\
\text { (nach Röntgendiagrammen) }\end{array}$ & 27 & SchMITT et al. ${ }^{9}$ & 32,5 \\
\hline $\begin{array}{l}\text { Quergestreifte Muskeln, Frosch, A-Zone, } \\
\text { Untereinheit aus dickeren Filamenten }\end{array}$ & 30 & SJÖSTRAND und ANDERSSON 18, 19 & 32,5 \\
\hline Quergestreifte Muskeln, Frosch, I-Zone & 40 & dieselben & 32,5 \\
\hline Glatte Muskeln, Uterus Ratte & 60 & GANSLER $^{11}$ & 65 \\
\hline $\begin{array}{l}\text { Herz- und Skelettmuskel, embryonal, Huhn, } \\
\text { Krabbe }\end{array}$ & $60-70$ & SPIRO $^{20}$ & 65 \\
\hline $\begin{array}{l}\text { Quergestreifte Muskeln, A-Zone, nicht } \\
\text { kontrahiert, Frosch }\end{array}$ & 60 & SJöSTRAND und ANDERSSON ${ }^{18,19}$ & 65 \\
\hline $\begin{array}{l}\text { Quergestreifte Muskeln, A-Zone, } \\
\text { verschiedene Tiere }\end{array}$ & 120 & HODGE $^{21}$ & 130 \\
\hline $\begin{array}{l}\text { Quergestreifte Muskeln, A-Zone, } \\
\text { kontrahiert }\end{array}$ & 140 & SJöstrand und ANDERSSON 18,19 & 130 \\
\hline Glatte Muskeln, Helix & 130 & diese Arbeit & 130 \\
\hline ebenso & 190 & diese Arbeit & 185 \\
\hline ebenso & 280 & SCHLOTE ${ }^{15}$ & 260 \\
\hline Glatte Muskeln, Regenwurm & 250 & HANSON $^{12}$ & 260 \\
\hline
\end{tabular}

Tab. 1. Verdopplungsreihen im Durchmesser von Myofilamenten aus glatten und quergestreiften Muskeln. Die „Sollwerte“ rechts stellen eine exakte geometrische Reihe mit der Steigung 2 dar. Ihr Anfangsglied ist so gewählt, daß die Abweichungen von den Meßwerten ein Minimum bilden. Die Meßwerte der mittleren Spalte geben die tatsächlich gefundenen oder beschriebenen Werte an. Über den aus der Reihe springenden Wert von $185 \AA$ vgl. Text.

\section{d) Deutung}

Eine Deutung der Tab. 1 setzt voraus, daß es auch vom physiologischen Standpunkt her erlaubt ist, glatte und quergestreifte Muskeln nebeneinander zu stellen. Ganze glatte Muskeln lassen sich in dieser Hinsicht nicht mit quergestreiften vergleichen. Sie leiten mit Dekrement ${ }^{21 a}$ (Schmaudt und Sleater 1955), besitzen viskosoiden Tonus ${ }^{22}$ (Jordan 1929) und können Sperrtonus erzeugen. Eine Erklärung für diese Verschiedenheit dürfte sein, daß glatte Muskeln überall im Inneren verstreut Ganglien-

* Hodge ${ }^{10}$ et al. finden an Querschnitten durch den Adductor von Venus Filamentdicken von etwa 200 bis $1000 \AA$. Da hierin jedoch Paramyosin mit enthalten sein kann, soll auf diese Daten nicht näher eingegangen werden.

21a W. Schmaudt u. W. Sleator JR., J. cellular comparat. Physiol. 46, 439 [1955].
Extrakte glatter Muskulatur dagegen verhalten sich unter ATP wie Extrakte quergestreifter Muskulatur ${ }^{5}$ (Ulbrecht 1952), nur daß die Dehnbarkeit des Aktomyosinkomplexes größer ist. Hiernach scheint es erlaubt, zwar nicht die ganzen Muskeln, wohl aber die Myofilamente von glatter und quergestreifter Muskulatur miteinander zu vergleichen. Sieht man von der verschiedenen Dehnbarkeit sowie vom wesentlich langsameren Verlauf einer Einzelzuckung ${ }^{22 a}$ (BozLeR 1930/31) ab, dann dürfte das sich vom quergestreiften Muskel unterscheidende Verhalten des ganzen glatten Muskels auf ganglionäre Elemente zurückführbar

22 H. J. Jordan, Allgemeine Vergleichende Physiologie der Tiere. W. de Gruyter und Co., Berlin 1929.

22a E. Bozler, Z. Vergleich. Physiol. 12, 579 [1930]; 14, 429 [1931]. 
sein, ja, es erscheint lohnend, in ihm außer motorischen Nervenendigungen auch sensible zu suchen.

Morphologisch gibt es manche Ähnlichkeiten zwischen den Myofilamenten der beiden Muskeltypen. Nach der Tab. 1 sind in glatten wie quergestreiften Muskeln als dünnste Filamenteinheiten Fäden von etwa $30 \AA$ Dicke teils gemessen teils errechnet worden. Beide Zellarten können außerdem auch Filamente von 60 und $120 \AA$ Durchmesser besitzen. Noch dickere Filamente sind bisher nur aus glatten Muskelzellen bekannt. In glatten wie in quergestreiften Muskeln ist auf Längsschnitten durch Filamentgruppen eine Querstreifung von etwa $250 \AA$ zu erkennen. $\left(90-250 \AA\right.$ am Frosch ${ }^{18,19}$ nach Suöstrand und Andersson 1956, 250-400 $\AA$ an Dipteren ${ }^{21}$ nach Hodge (1956), $220 \pm 30 \AA$ an teilkontrahierten glatten Muskeln von Helix in der vorliegenden Arbeit). Der Flächenanteil, den die Filamente an einem Querschnitt durch ein Myofilamentbündel nehmen, ist für beide Muskelarten größenordnungsmäßig ebenfalls vergleichbar.

Betrachtet man nunmehr an Hand der Tab. 1 die Filamentdurchmesser in den einzelnen Gewebe- und Tierarten, so fällt auf, daß sich rein formal die dikkeren Filamente durch Verdopplung des Durchmessers aus den nächstdünneren herleiten lassen. Würde eine genaue Verdopplung vorliegen, dann müßte sich für die verschiedenen Durchmesser eine geometrische Reihe mit der Steigung 2 aufstellen lassen. Eine solche Reihe ist auf der rechten Seite der Tab. 1 („Sollwerte“) aufgestellt. Berücksichtigt man die Streuung der Meßwerte, präparativ bedingte Abweichungen, ferner die Schwierigkeiten, welche bei der Eichung von Elektronenmikroskopen auf mit hoher Genauigkeit reproduzierbare Vergrößerungswerte auftreten, schließlich die Tatsache, daß der Durchmesser der Myofilamente sich aus Gründen mangelnden Kontrastes besonders an Skelettmuskeln schwer exakt fassen läßt, dann ist die Übereinstimmung zwischen gemessenen und in eine Verdopplungsreihe geordneten Werten sehr befriedigend. Die Abweichung beträgt nirgends mehr als 10 Prozent. Lediglich der 190-Å-Wert bildet eine Ausnahme.

Es ist nützlich, sich über die mögliche Bedeutung dieser Reihe klar zu werden. Wenn Kreisdurchmesser gesetzmäßig von Stufe zu Stufe um das Doppelte zunehmen, dann wächst ihre Fläche jeweils um das Vierfache. Würde der Zuwachs an Durchmesser

23 L. Pauling u. R. B. Correx, Nature [New York] 171, 59 [1953]. das $\sqrt{2}$-fache betragen, so stiege die Kreisfläche auf das Doppelte. Der dünnste Faden aus der Tab. 1 hat einen Durchmesser von $10-20 \AA$. Der Sollwert beträgt, sofern eine echte Verdopplungsreihe vorliegt, 16,25 A. Danach könnte es sein, daß Myofilamente von 32,$5 ; 65 ; 130 ; 260 \AA$ Dicke sich aus $4 ; 16 ; 64 ; 256$ eng beieinanderliegenden dünnsten Einheiten zusammensetzen.

Zwar ist in molekularen Bereichen ein solcher Packungsgrad wohl denkbar. Die Vorstellung, daß ein Myofilament ausschließlich als ein Strang von einander parallelen Fadenmolekülen aufzufassen sei, befriedigt aus mechanischen Gründen jedoch nicht und verträgt sich auch nicht mit den verschiedenen Dicken der Filamente in A- und I-Zone quergestreifter Muskeln.

Die Verdopplungsreihe der Filamentdurchmesser kann auch anders gedeutet werden. Sıöstrand und Andersson 18, 19 (1956) nehmen an, daß die dickeren Myofilamente quergestreifter Muskeln eine mehrfach gewundene Schraube darstellen, deren dünnste Einheit etwa $30 \AA$ dick ist (Sollwert der Tab. 1 $32,5 \AA$ ). Jedoch kann ein Filament von etwa $30 \AA$ Durchmesser noch nicht letzte makromolekulare Einheit sein, da Eiweiß-Fadenmoleküle, die nach $\mathrm{P}_{\mathrm{AU}}$ LING und CoRey ${ }^{23}$ (1953) als $\alpha$-Helix gebaut sind, kaum dicker als $15 \AA$ sein dürften.

Man weiß, daß zwei Komponenten zusammentreten müssen, um eine sich mittels ATP kontrahierende Faser $\mathrm{zu}$ erzeugen, Aktin und Myosin ${ }^{\mathbf{2 4}}$ (Zusammenfassung bei Weber 1956). Ist die Hypothese vom Schraubenbau zutreffend, dann können beide Substanzen, verbunden als Aktomyosin, schwerlich anders als gemeinsam in der dünnsten funktionellen Einheit enthalten sein. Hinweise dafür fanden Sэöstrand und Andersson ${ }^{18,19}$, als sie feststellten, daß ein stark gedehntes Myofilament sich bei ihnen in drei Fäden zu je 10 bis $20 \AA$ A Dicke aufspaltet.

$\mathrm{Ob}$ es immer 3 Fäden sein müssen, worauf die Dreiecksform ihrer Myofilamente zunächst hindeuten würde, ob man, da sich im quergestreiften Muskel die Mengen von Myosin zu Aktin wie etwa 3:1 verhalten, nicht vier Fäden fordern muß, dies mag zunächst dahingestellt bleiben. Auf alle Fälle ist ein 30-Å-Faden noch nicht letzte makromolekulare Einheit, sondern er besteht aus mehreren Untereinheiten zu je 10-20 Dicke (Sollwert nach Tab. 16, $3 \AA \AA$ ), die, wie S.östrand und Andersson ausführen, wohl Eiweißfaden-Moleküle darstellen müssen.

Denkt man sich aus dem 30-Å-Faden (Sollwert $32,5 \AA$ ) eine Schraube in der Form einer Wendel-

24 H. H. Weber, Fortschr. Zool. (M. Hartmans), 10, 304 [1956]. 
treppe gewickelt, dann müßte sie einen Außendurchmesser von $2 \cdot 32,5 \AA=65 \AA$ besitzen. Läßt man sich diese 65 - $\AA$-Schraube ihrerseits abermals eng um eine Achse herumwinden, dann entsteht eine Doppelschraube mit $130 \AA$ Außendurchmesser und so fort. Auch auf diese Weise könnte man also die Verdopplungsreihe der Myofilament-Durchmesser verstehen. Kleine Torsionen der Primärschraube vermöchten dann, große Längenänderungen des dicken Myofilamentes hervorzurufen und die beträchtliche Dehnbarkeit glatter Muskeln hätte ihren Grund in dem hohen Aufschraubungsgrad ihrer Myofilamente gefunden.

Offen bleibt, wie ein Filament-Durchmesser von $190 \AA$ verstanden werden muß. Er paßt nicht zu den Vorstellungen von mehrfacher Aufschraubung einer 32,5 $\AA$ dicken Einheit. Myofilamente dieser Stärke bestehen nach dem oben gesagten aus drei, vielleicht vier Eiweiß-Fadenmolekülen. Da Muskeln nur zwei makromolekulare Substanzen enthalten, Aktin und Myosin, liegt es nahe, anzunehmen, daß zwei Fadenmoleküle auch genügen würden, um eine funktionelle Einheit zu bilden. Dies ergäbe einen dünnsten Faden von $16,25 \cdot \sqrt{2}=23 \AA$ Durchmesser. Eine dreifache Aufschraubung dieses hypothetischen 23-Å-Filaments müßte Myofilamente von $184 \AA$ Dicke ergeben. Danach sollte dieser glatte Muskel von Helix also Myofilamente unterschiedlicher Zusammensetzung enthalten. Die Bestätigung der Folgerung an Hand hochaufgelöster Elektronenbilder und chemischer Analysen auf Aktinund Myosingehalt bleibt abzuwarten.

Von ganz anderer Seite her gibt es bemerkenswerte Parallelen, welche zeigen, daß Doppelfäden oder Fäden noch höherer Ordnung durchaus vorkommen können. Vergleicht man nämlich, wie an anderem Ort berichtet werden soll, die Dicken der bisher gemessenen Chromonemata aus den verschiedensten Organismen miteinander 25, 26 (für Bakterien vgl. Preusser, Schlote, RipPEL 1957; Preusser 1957), so kommt man ebenfalls zu einer Zahlenreihe. Ihre Steigung beträgt aber nicht 2,

25 H. J. Preusser, Fr. W. Schlote u. A. Rippel-Baldes, Naturwissenschaften 44, 186 [1957].

26 H. J. Preusser, Dissertation, Göttingen 1957.

27 J.D. Watson u. F. H. C. Crick, Cold Spring Harbor Sympos. quantitat. Biol. 18, 123 [1953].

28 E. De Robertis, J. Biophysic. and Biochem. Cytol. 2, 785 [1956]. sondern $\sqrt{2}$ ! Das Chromonema sollte danach in sich polynem gebaut sein und müßte aus $2^{n}(n=0 ; 1$; 2...) einander und der Längsachse des Fadens parallelen dünnsten Einheiten bestehen (Sollwert etwa $19 \AA$, vgl. das DNS-Modell ${ }^{27}$ von W $_{\text {ATson }}$ und CRICK 1953 mit $20 \AA ̊$ Dicke). Soweit Dicken bisher an Dünnschnitten gemessen wurden, reichen sie von $47 \AA^{28}$ (DE RoBERtis 1956) bis etwa $200 \AA \AA^{25,} 29$ (SJöstrand und Afzelius 1956, Preusser, Schlote, Rippel 1957). Die Sollwerte betragen unter Zugrundelegung einer geometrischen Reihe mit der Steigung $\sqrt{2}: 53 \AA$ und $220 \AA$. . Ein Schraubenbau wurde innerhalb dieser Dicken bisher nie gefunden. Sie ist bei einer Steigung der Reihe um $\sqrt{2}$ auch nicht zu erwarten.

Eine andere Deutung der Tatsache, daß sich Myofilamente, deren Durchmesser um $\sqrt{2}$ voneinander verschieden ist (130 und $190 \AA)$, nebeneinander in derselben Muskelzelle finden, ist zur Zeit nicht möglich, will man nicht annehmen, daß die Kontraktion der Myofilamente von einem gleichförmigen Anwachsen ihrer Durchmesser begleitet ist und also alle Werte der Tab. 1 sich nur rein zufällig zu einer geometrischen Reihe zusammenfügen.

Die Alternative dazu ist, daß es im Verlauf einer Kontraktion Bedingungen gibt, unter denen ein Filament-Durchmesser sich verdoppelt, weil das Filament in den nächsthöheren Aufschraubungs-Zustand übergeht. Sprunghaft steigende Dicken bei stetig zunehmendem Kontraktionszustand wären die Folge. Die Tatsache, daß aus den Angaben der Literatur und eigenen Befunden eine Reihe von sich jeweils verdoppelnden oder doch gesetzmäßig vergrößernden Filament-Durchmessern ohne weiteres aufgestellt werden kann, ist ein gutes Argument für die Schrauben-Hypothese. Durch ihr hohes reversibles Dehnungsverhältnis werden glatte Muskeln ungleich geeigneter sein, die Frage zu lösen, als quergestreifte.

Das Kontraktionsmodell von Huxley und Hanson 30 (1956) wie das Strukturmodell von Hodge ${ }^{31}$ (1955) wurden hier nicht berührt, weil weder die Verhältnisse im glatten Muskel noch die vorliegenden Zahlenreihen dazu Veranlassung gaben.

29 F. S. Sü̈strand u. B. A. Afzelius, Electron Microscopy, Proc. Stockholm Conf. 164, Almqvist and Wiksell, Stockholm 1956.

30 H. E. Huxley u. J. Hanson, Electron Microscopy, Proc. Stockholm Conf. 202, Almqvist and Wiksell, Stockholm 1956.

31 A. J. Hodge, J. Biophysic. and Biochem. Cytol. 1, 361 [1955]. 